www.nature.com/ejhg

\title{
Identification of novel SDHD mutations in patients with phaeochromocytoma and/or paraganglioma
}

\author{
Alberto Cascon $^{\star}{ }^{1}$, Sergio Ruiz-Llorente ${ }^{1}$, Arancha Cebrian ${ }^{1}$, Dolores Telleria ${ }^{1}$, \\ Jose Carlos Rivero ${ }^{1}$, Juan Jose Diez ${ }^{2}$, Pablo J Lopez-Ibarra ${ }^{3}$, Miguel Angel Jaunsolo ${ }^{4}$, \\ Javier Benitez ${ }^{1}$ and Mercedes Robledo ${ }^{1}$
}

${ }^{1}$ Department of Human Genetics, Spanish National Cancer Center (CNIO), Madrid, Spain; ${ }^{2}$ Endocrinology Service, Hospital Ramon y Cajal, Madrid, Spain; ${ }^{3}$ Endocrinology Service, Hospital Universitario San Cecilio, Granada, Spain; ${ }^{4}$ Endocrinology and Nutrition Service, Hospital Severo Ochoa, Madrid, Spain

Familial paraganglioma is a dominantly inherited disorder characterised by the development of highly vascular tumours in the head and neck. Recently, a relationship between hereditary tumours derived from the autonomic nervous system and germline mutations in the gene encoding succinate dehydrogenase complex subunit $\mathrm{D}(S D H D)$ is increasingly a subject of study. Familial paraganglioma syndrome is embryologically related to phaeochromocytoma, another neuroendocrine tumour that shows great aetiological and genetic heterogeneity. Some hereditary phaeochromocytomas may be associated with germline mutations in VHL, RET and NF1 genes in genetic disorders such as von Hippel-Lindau disease (VHL), multiple endocrine neoplasia type 2 (MEN 2) and neurofibromatosis type 1 (NF 1), respectively. However, there are many cases that cannot be explained by mutations in these genes. In this report, we describe two previously unreported mutations in two patients from 25 unrelated kindreds with phaeochromocytoma and/or paraganglioma disorders and with or without familial antecedents: a mutation featuring the change of tryptophan to a termination codon in exon 2, and a 4-bp deletion in exon 4 that results in a truncated protein. We also describe one missense substitution of uncertain significance. The patients had previously tested negative for germline mutations in VHL and RET genes and had not been previously selected. The involvement of SDHD mutations in familial phaeochromocytoma and/or paraganglioma predisposition is of considerable interest since other studies have shown these alterations to be associated with highly expressed angiogenic factors.

European Journal of Human Genetics (2002) 10, 457-461. doi:10.1038/sj.ejhg.5200829

Keywords: phaeochromocytoma; paraganglioma; SDHD; germline mutation

\section{Introduction}

Hereditary paraganglioma is a dominantly inherited disorder characterised by vascular tumours arising in extraadrenal nonchromaffin tissue. Even though paragangliomas appear in the head and the neck, the most frequent location is the carotid body, the organ that senses oxygen levels in blood. ${ }^{1}$ Penetrance of this disease is incomplete when transmitted through mothers since

*Correspondence: Alberto Cascon, Department of Human Genetics, Centro Nacional de Investigaciones Oncologicas, Melchor Fernandez Almagro 3, 28029 Madrid, Spain. Tel: 34-91-224 69 47;

Fax: 34-91-224 69 23; E-mail: acascon@cnio.es

Received 5 February 2002; revised 23 April 2002; accepted 25 April 2002 children of affected mothers rarely, if ever, develop the disease, suggesting transmission with maternal imprinting. $^{2}$

Recent analyses of familial paragangliomas revealed some cases with germline mutations in the $S D H D$ gene located on chromosome $11 \mathrm{q} 23^{2-6}$ as well as in the $S D H B$ gene located on chromosome band $1 \mathrm{p} 35-36.1 .^{7}$ The SDHD gene is the subject of exhaustive study because it has been postulated that the mitochondrial electron transport chain plays a role in oxygen sensing and signalling. ${ }^{8}$ Recent studies have concluded that inactivation of SDHD gene in hereditary paraganglioma by means of germline mutations leads to the complete loss of mitochondrial complex II activity 
and stimulates a high level of expression of angiogenic factors, which activate the hypoxia pathway. ${ }^{9}$

Paragangliomas arising from the parasympathetic ganglia share a similar embryological origin with pheochromocytomas. $^{10,11}$ Phaeochromocytoma is a neuroendocrine chromaffin-staining tumour that usually causes secondary hypertension by oversecretion of catecholamines. ${ }^{1}$ Recent advances in molecular genetics are revealing the nature of the pathogenesis of phaeochromocytoma, with the reporting of multiple genetic alterations. Most phaeochromocytomas are sporadic, but approximately $10 \%$ are hereditary and may be found in association with von Hippel-Lindau disease, multiple-endocrine neoplasia type 2 or neurofibromatosis type $1 .^{11-13}$ However, the aetiology of most familial and sporadic forms of phaeochromocytoma is still unknown and remains to be characterised.

Since the discovery of germline mutations of SDHD in some patients with hereditary paraganglioma, ${ }^{2}$ several authors have tried to determine whether this gene plays a role in the development of neuroendocrine tumours. ${ }^{14}$ Recently, Gimm et al. have found germline SDHD mutations in approximately $11 \%$ of patients with sporadic phaeochromocytoma, suggesting an involvement of this gene with the disease. ${ }^{15}$

In the present study we have looked for new mutations in the SDHD gene and thereby to establish whether the alterations of this suppressor gene are definitely linked to the pathogenesis of tumours derived from the autonomic nervous system and its role in the susceptibility to developing phaeochromocytoma and/or not only familial, but also sporadic, pararaganglioma.

\section{Materials and methods Patients}

The SDHD mutation analysis was performed in 25 consecutive patients, without previous selection, with phaeochromocytoma and/or paraganglioma (Table 1), 11 to 68 years of age, with or without familial antecedents and from unrelated families who tested negative for germline mutations in VHL and RET genes (unpublished data). We used DNA from 140 unrelated and unaffected individuals as a control population. Genomic DNA was extracted from the patients' blood samples following a standard method. ${ }^{16}$ Informed consent was obtained from all patients.

\section{Amplification and sequencing analysis}

The analysis was carried out by genomic DNA amplification of peripheral blood leukocytes. SDHD comprises three introns and four exons. Exon-specific polymerase chain reaction (PCR) and direct sequencing analyses were performed in exons 1 to 4 of the SDHD gene. The primer pairs for exon amplification were designed on the basis of the genomic sequences (accession number AB026906) and were as follows: 1F (5'-ATT GTC GCC TAA GTC CTT CC3'), 1R (5'-CTG GAG GCT ACG CTA AGC AC-3'), 2F (5'TCA GTC CTG TTA AAG GAG AGG TTC-3'), 2R (5'-TAG

Table 1 Clinical and molecular data of the patients analysed

\begin{tabular}{|c|c|c|c|c|c|}
\hline Patient ID & Age at onset/sex & Paraganglioma & Pheochromocytoma & Nucleotide change & Amino acid change \\
\hline 1 & $62 / \mathrm{m}$ & - & Unilateral & - & - \\
\hline 2 & $68 / \mathrm{m}$ & - & Unilateral & - & - \\
\hline 3 & $36 / f$ & - & Unilateral & - & - \\
\hline 4 & $34 / f$ & - & Unilateral & - & - \\
\hline 5 & $62 / f$ & - & Unilateral & - & - \\
\hline 6 & $22 / \mathrm{m}$ & - & Bilateral & - & - \\
\hline 7 & $36 / f$ & - & Bilateral & - & - \\
\hline 8 & $51 / f$ & - & Unilateral & - & - \\
\hline 9 & $38 / f$ & - & Bilateral & $\mathrm{AGC} \rightarrow \mathrm{AGT}$ & S68S \\
\hline 10 & $42 / f$ & - & Unilateral & - & - \\
\hline 11 & $11 / \mathrm{m}$ & - & Unilateral & - & - \\
\hline 12 & $42 / f$ & - & Unilateral & - & - \\
\hline 13 & $40 / \mathrm{m}$ & - & Unilateral & $\begin{array}{l}\mathrm{GGT} \rightarrow \mathrm{AGT} \\
\mathrm{AGC} \rightarrow \mathrm{AGT}\end{array}$ & $\begin{array}{l}\text { G12S } \\
\text { S68S }\end{array}$ \\
\hline 14 & $49 / \mathrm{m}$ & - & Unilateral & - & - \\
\hline 15 & $41 / \mathrm{m}$ & - & Unilateral & $\mathrm{CAC} \rightarrow \mathrm{CGC}$ & H50R \\
\hline 16 & $37 / \mathrm{m}$ & - & Bilateral & - & - \\
\hline 17 & $14 / f^{*}$ & - & Unilateral & - & - \\
\hline 18 & $48 / \mathrm{m}$ & - & Bilateral & - & - \\
\hline 19 & $40 / \mathrm{m}^{*}$ & Paraaortic and carotid & - & $\mathrm{TGG} \rightarrow \mathrm{TGA}$ & W43X \\
\hline 20 & $22 / f$ & Paraaortic & - & - & - \\
\hline 21 & $30 / \mathrm{m}$ & Abdominal & - & - & - \\
\hline 22 & $36 / f$ & Head & - & - & - \\
\hline 23 & $22 / f$ & Paraaortic & Unilateral & - & - \\
\hline 24 & $20 / f$ & Paraaortic and bilateral carotid & Unilateral & 13732delGACT & - \\
\hline 25 & $32 / \mathrm{m}^{*}$ & Paraaortic & Unilateral & - & - \\
\hline
\end{tabular}

*Patients with familial antecedents 
AGC CCA GAA AGC AGC AG-3'), 3F (TTT GGG TTA CAG TGT GGC ATA-3'), 3R (5'-CAC AGC AAA CAA ACT GAG CA-3'), 4F (5'-GTC TTC TAA TTT CAC TGT GGT TTT T-3'), 4R (5'-TTC AAA GTA TGA AGT CAA AAA GGT C-3'). PCR was performed with Gene Amp PCR System 9700 thermocycler (Perkin Elmer, USA) according to the manufacturer's instructions. The purified products were subsequently sequenced using an automatic sequencer ABI PRISM ${ }^{\mathrm{TM}}$ 3700 (Applied Biosystems. Perkin Elmer, USA).

\section{Single Strand Conformation Polymorphism (SSCP) and} enzyme analysis

SSCP analysis was performed as previously described. ${ }^{17}$ PCR products displaying mobility shift were subsequently sequenced. The enzyme analysis was performed by digesting amplified PCR products with $5 \mathrm{u}$ of TspRI endonuclease and the digestion products were analysed by $3 \%$ agarose gel electrophoresis to detect the nucleotide change.

\section{Results}

We screened 25 patients with phaeochromocytoma and/or paraganglioma tumours for the presence of germline mutations in SDHD gene by amplification analysis followed by sequencing of PCR products. We identified five heterozygous variants (2/7 paragangliomas and $3 / 18$ phaeochromocytomas): one nonsense mutation, two missense substitutions, one silent mutation and one deletion (Table 1).

We found a transition GGT $\rightarrow$ AGT (G12S) in exon 1 of a 40year-old male (case 13) with a surgically removed phaeochromocytoma and no family history. This G12S transition resulted in the creation of a TspRI restriction site found in five of $200(2.5 \%)$ chromosomes of the control population used in the study by means of the enzyme assay designed to screen for this change. The same patient who showed variant G12S had a silent mutation AGC $\rightarrow$ AGT (S68S) in exon 3. S68S also occurred in a 38-year-old female with bilateral phaeochromocytoma and no family history (case 9).

Another transition, CAC $\rightarrow$ CGC (H50R), was found in exon 2 of a 42-year-old male (case 15) with an adrenal phaeochromocytoma that had been surgically removed by laparoscopic adrenalectomy (Figure 1). This missense substitution was found in four of the 280 chromosomes tested by SSCP analysis (1.4\%). This patient had no family history and tumour samples were not available to perform $\mathrm{LOH}$ analysis to confirm the nature of the change.

A 4-bp frameshift deletion in codon 112 (13732delGACT) was detected in exon 4 of a 20-year-old female (case 24). This individual had undergone removal of adrenal phaeochromocytoma and paraaortic paraganglioma, and had bilateral carotid glomus antecedents. No apparent family history was reported for this 20-year-old patient. This unreported frameshift deletion gave rise to a 132-amino acidtruncated protein by creating a premature stop codon.

The second mutation found in this study was a transition, TGG $\rightarrow$ TGA (W43X), at nucleotide 129 in exon 2

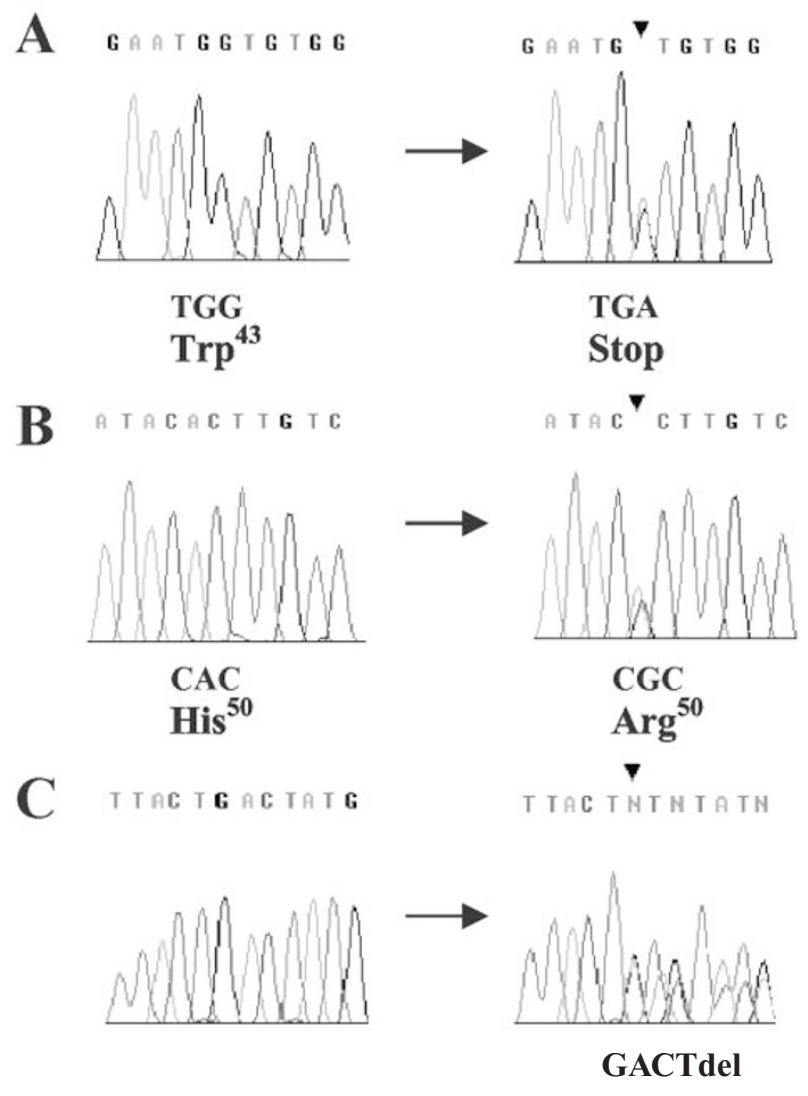

Figure 1 Sequencing chromatograms showing alterations (denoted with one arrowhead). Affected codons and aminoacids are indicated below.

(case 19). This new nonsense mutation of the SDHD gene changes a tryptophan to a premature termination codon resulting in a truncated SDHD of 42 aminoacids. The patient with this nonsense mutation was a 40 -year-old male when he was admitted to hospital and was the only one with a familial history of phaeochromocytoma. The patient's father died after a secondary hypertensive crisis due to bilateral phaeochromocytoma, and a paternal aunt suffered hypertension and paraganglioma. The patient showed paraaortic paraganglioma and had had two carotid paragaglioma surgically removed.

\section{Discussion}

Molecular analysis of the entire coding region of RET and VHL genes (and even MEN1) to find mutations is the commonest method used in paraganglioma and phaeochromocytoma diagnosis, but currently the alterations found in these genes alone cannot explain the development of such tumours.

Germline mutations in SDHD have been recently reported as causing hereditary and nonfamilial paraganglioma, implying a tumour suppressor role for SDHD. ${ }^{18}$ Linkage between SDHD mutations and familial phaeochromocyto- 
ma also seems to be a possible mechanism for tumour susceptibility, and so inactivating mutations of the SDHD gene appear to be of considerable interest in individuals with familial, multiple, or early-onset phaeochromocytomas and even in nonfamilial phaeochromocytoma. ${ }^{15,19}$ However, to date there are few available data on this matter and this aspect needs further evaluation.

SDHD maps to chromosome band 11q23 and encodes succinate dehydrogenase complex subunit $\mathrm{D}$, the small subunit of cytochrome $b$ in complex II (succinate-ubiquinone oxidoreductase). ${ }^{20}$ Complex II shares important funtions in both the tricarboxylic acid cycle and the aerobic electron transport chain of mitochondria and constitutes the only direct link between activity in the citric acid cycle and electron transport in the membrane. ${ }^{21}$ SDHD seems to be essential for the interaction between the complex and quinone species. ${ }^{22}$

So, the inactivation of SDHD gene in hereditary paraganglioma leads to a complete loss of mitochondrial complex II activity. ${ }^{9}$ This mechanism disrupts the hypoxia pathway by means of chronic hypoxic stimulation and cellular proliferation and could be responsible for some cases of paraganglioma and phaeochromocytoma tumours.

In this study, we searched for mutations in the SDHD gene in 25 patients with phaeochromocytoma (18 cases), paraganglioma (four cases) alone, and with both (four cases). The patients, with or without familial antecedents and without previous selection, were previously analysed for VHL and RET mutations. Among the samples examined we identified five germline SDHD variants distributed among the four exons of the gene: G12S, S68S, H50R, W43X and 13732delGACT. Gimm et al. found G12S change in approximately $1.3 \%$ of their control population, and they postulated that this variant was either a pathogenic and low-penetrance mutation or a very rare polymorphism. ${ }^{15}$ G12S variant changes not only the glycine residue of the sequence GALGGR of SDHD protein but also, therefore, a putative sequence of $\mathrm{N}$-myristoylation that could be important in proteolytic processing. ${ }^{23}$ However, the presence of this change in $2.5 \%$ of the control population led us to conclude that this change constitutes a polymorphism.

The S68S variant was also found in all the five controls that tested positive for G12S change and in a patient with spinal paraganglioma, ${ }^{4}$ so we can conclude that these variants are in linkage disequilibrium.

The H50R missense substitution found in exon 2 causes an amino acid change that could alter the protein conformation. Despite both aminoacids being hydrophilically charged, arginine is more positively charged than histidine under neutral conditions. ${ }^{24}$ Histidine 50 is located in a zone of the potential transit peptide of the protein that is conserved in human, bovine and murine SDHD. LOH and segregation analyses were not carried out since neither tumour samples of patients or relative's samples were available. The SSCP analysis revealed the arginine variant in four of 280 control chromosomes (1.4\%) so we can postulate either that this is a change of uncertain significance or a rare polymorphism. Further studies will be necessary to clarify the importance of this substitution.

The two mutations, 13732delGACT and W43X, found in exons 2 and 4 respectively, both yield a truncated protein. The 4-bp frameshift deletion caused a truncated protein of 132 aminoacids by creating a premature stop codon, which lacked part of the second transmembrane domain of the protein. This mutation appeared in a 20 -year-old female with several tumours derived from the sympathetic nervous system. The change was found in a suggested mutational hot $\operatorname{spot}^{4}$ located within the same five nucleotides where three different alterations (1-bp insertion, a transition and our 4-bp deletion) have been found.

The W43X nonsense mutation created a premature termination codon resulting in a truncated SDHD of 42 aminoacids that lacked the transmembrane, signal and heme-binding domains. This new germline mutation affected a 40-year-old patient with paraaortic paraganglioma and two operated carotid paraganglioma and a familial history of phaeochromocytoma. Gimenez-Roqueplo et al. described a nonsense mutation (R22X) featuring a complete loss of complex II enzymatic activity in inherited phaeochromocytoma not detected in sporadic phaeochromocytomas. ${ }^{9}$ We can assume that the same loss has occurred in the W43X mutation.

The identification of new mutations in genes like SDHD could represent a new approach to the determination of paraganglioma and phaeochromocytoma susceptibility. Presymptomatic diagnosis of at risk individuals can be carried out by molecular analysis of this gene, and perhaps these novel SDHD mutations could help us determine whether the relationship between the respiratory chain and oxygen sensing may be considered to be a mechanism for tumour susceptibility.

In summary, despite it having been reported that complex II deficiency is a rare condition in humans, ${ }^{25}$ we have described two mutations in SDHD (W43X, 13732delGACT) that resulted in complex II inactivity in $2 / 7$ patients with paraganglioma, one of them also with pheochromocytoma. We have also found one missense variant of uncertain significance and two polymorphisms in linkage desequilibrium in patients with phaeochromocytoma.

The results obtained in this study raise the possibility of applying this approach as a routine genetic screening in patients who test negative for germline mutations in other genes associated pathologies such as RET or VHL.

\footnotetext{
Acknowledgements

We thank the patients participating in this study and the clinicians who provided blood samples and clinical information. We are also grateful for the technical assistance of Rocio Letón. This work was partially supported by the Fondo de Investigaciones Sanitarias project FIS 00/0118. Alberto Cascón is a postdoctoral fellow of the Excmo. Ayuntamiento de Madrid.
} 


\section{References}

1 Koch CA, Vortmeyer AO, Huang SC, Alesci S, Zhuang Z, Pacak K: Genetic aspects of pheochromocytoma. Endocr Regulations 2001; 35: $43-52$.

2 Baysal BE, Ferrell RE, Willett-Brozick JE et al: Mutations in SDHD, a Mitochondrial Complex II Gene, in Hereditary Paraganglioma. Science 2000; 287: 848-851.

3 Badenhop RF, Cherian S, Lord RS, Baysal BE, Taschner PE, Schofield PR: Novel mutations in the SDHD gene in pedigrees with familial carotid body paraganglioma and sensorineural hearing loss. Genes Chromosomes Cancer 2001; 3: 255-263.

4 Masuoka J, Brandner S, Paulus W et al: Germline SDHD mutation in paraganglioma of the spinal cord. Oncogene 2001; 20: $5084-$ 5086.

5 Milunsky JM, Maher TA, Michels VV, Milunsky A: Novel mutations and the emergence of a common mutation in the SDHD gene causing familial paraganglioma. Am J Med Genet 2001; 100: $311-314$

6 Taschner PE, Jansen JC, Baysal BE et al: Nearly all hereditary paragangliomas in the Netherlands are caused by two founder mutations in the SDHD gene. Genes Chromosomes Cancer 2001; 31: 274-281.

7 Astuti D, Latif F, Dallol A, Dahia PL, Douglas F, George E, Skoldberg F, Husebye ES, Eng C, Maher ER: Gene mutations in the succinate dehydrogenase subunit SDHB cause susceptibility to familial pheochromocytoma and to familial paraganglioma. Am J Hum Genet 2001; 69: 49-54.

8 Chandel NS, Maltepe E, Goldwasser E, Mathieu CE, Simon MC, Schumacker PT: Mitochondrial reactive oxygen species trigger hypoxia-induced transcription. Proc Natl Acad Sci USA 1998; 95: $11715-11720$

9 Gimenez-Roqueplo AP, Favier J, Rustin P et al: The R22X mutation of the SDHD gene in hereditary paraganglioma abolishes the enzymatic activity of complex II in the mitochondrial respiratory chain and activates the hypoxia pathway. Am J Hum Genet 2001; 69: $1186-1197$.

10 Milunsky J, DeStefano AL, Huang XL et al: Familial paragangliomas: linkage to chromosome 11q23 and clinical implications. Am J Med Genet 1997; 72: 66-70.

11 Anderson RJ, Lynch HT: Familial risk for neuroendocrine tumors. Curr Opin Oncol 1993; 5: 75-84.

12 Schimke RN: Multiple endocrine neoplasia: how many syndromes? Am J Med Genet 1990; 37: 375 - 383.

13 Maher ER, Webster AR, Richards FM et al: Phenotypic expression in von Hippel-Lindau disease: correlations with germline VHL gene mutations. J Med Genet 1996; 33: $328-332$.
14 Aguiar RC, Cox G, Pomeroy SL, Dahia PL: Analysis of the SDHD gene, the susceptibility gene for familial paraganglioma syndrome (PGL1), in pheochromocytomas. J Clin Endocrinol Metab 2001; 86: 2890-2894.

15 Gimm O, Armanios M, Dziema H, Neumann HP, Eng C: Somatic and occult germ-line mutations in SDHD, a mitochondrial complex II gene, in nonfamilial pheochromocytoma. Cancer Res 2000; 60: 6822-6825.

16 Sambrook J, Fritsch EF, Maniatis T: Molecular Cloning: a Laboratory Manual; 2nd edn. Cold Spring Harbor, NY: Cold Spring Harbor Laboratory Press, 1989.

17 Orita M, Suzuki Y, Sekiya T, Hayashi K: Rapid and sensitive detection of point mutations and DNA polymorphisms using the polymerase chain reaction. Genomics 1989; 5: 874-879.

18 Baysal BE, Willett-Brozick JE, Taschner PE, Dauwerse JG, Devilee P, Devlin B: A high-resolution integrated map spanning the SDHD gene at 11q23: a 1.1-Mb BAC contig, a partial transcript map and 15 new repeat polymorphisms in a tumour-suppressor region. Eur J Hum Genet 2001; 9: 121-129.

19 Astuti D, Douglas F, Lennard TW et al: Germline SDHD mutation in familial phaeochromocytoma. Lancet 2001; 357: 1181-1182.

20 Hirawake H, Taniwaki M, Tamura A, Kojima S, Kita K: Cytochrome $\mathrm{b}$ in human complex II (succinate-ubiquinone oxidoreductase): cDNA cloning of the components in liver mitochondria and chromosome assignment of the genes for the large (SDHC) and small (SDHD) subunits to 1q21 and 11q23. Cytogenet Cell Genet 1997; 79: 132-138.

21 Ackrell BAC: Progress in understanding structure-function relationships in respiratory chain complex II. FEBS Lett 2000; 466: $1-5$.

22 Shenoy SK, Yu L, Yu CA: The smallest membrane anchoring subunit (QPs3) of bovine heart mitochondrial succinate-ubiquinone reductase. Cloning, sequencing, topology, and Q-binding domain. J Biol Chem 1997; 272: 17867-17872.

23 Towler DA, Gordon JI, Adams SP, Glaser L: The biology and enzymology of eukaryotic protein acylation. Annu Rev Biochem 1988; 57: 69-99.

24 Yuasa H, Ito M, Oiso Y, Kurokawa M et al: Novel mutations in the V2 vasopressin receptor gene in two pedigrees with congenital nephrogenic diabetes insipidus. J Clin Endocrinol Metab 1994; 79: $361-365$.

25 Parfait B, Chretien D, Rotig A, Marsac C, Munnich A, Rustin P. Compound heterozygous mutations in the flavoprotein gene of the respiratory chain complex II in a patient with Leigh syndrome. Hum Genet 2000; 106: 236-243. 